\title{
Prospective Head Motion Correction Using FID-Guided On-Demand Image Navigators
}

\author{
Maryna Waszak, ${ }^{1,2,3 \dagger *}$ Pavel Falkovskiy, ${ }^{1,2,3 \dagger}$ Tom Hilbert, ${ }^{1,2,3}$ Guillaume Bonnier, ${ }^{1,2,3}$ \\ Bénédicte Maréchal, ${ }^{1,2,3}$ Reto Meuli, ${ }^{3}$ Rolf Gruetter, ${ }^{3,4,5}$ Tobias Kober, ${ }^{1,2,3}$ and \\ Gunnar Krueger ${ }^{2,3,6}$
}

Purpose: We suggest a motion correction concept that employs free-induction-decay (FID) navigator signals to continuously monitor motion and to guide the acquisition of image navigators for prospective motion correction following motion detection.

Methods: Motion causes out-of-range signal changes in FID time series that, and in this approach, initiate the acquisition of an image navigator. Co-registration of the image navigator to a reference provides rigid-body-motion parameters to facilitate prospective motion correction. Both FID and image navigator are integrated into a prototype magnetization-prepared rapid gradient-echo (MPRAGE) sequence. The performance of the method is investigated using image quality metrics and the consistency of brain volume measurements.

Results: Ten healthy subjects were scanned (a) while performing head movements (nodding, shaking, and moving in $z-$ direction) and (b) to assess the co-registration performance. Mean absolute errors of $0.27 \pm 0.38 \mathrm{~mm}$ and $0.19 \pm 0.24^{\circ}$ for translation and rotation parameters were measured. Image quality was qualitatively improved after correction. Significant improvements were observed in automated image quality measures and for most quantitative brain volume computations after correction.

Conclusion: The presented method provides high sensitivity to detect head motion while minimizing the time invested in acquiring navigator images. Limits of this implementation arise from temporal resolution to detect motion, false-positive alarms, and registration accuracy. Magn Reson Med 78:193-203, 2017. () 2016 International Society for Magnetic Resonance in Medicine

Key words: MRI; motion detection; FID navigators; head imaging; image navigators; prospective motion correction

\footnotetext{
${ }^{1}$ Advanced Clinical Imaging Technology (HC CMEA SUI DI BM PI), Siemens Healthcare AG, Lausanne, Switzerland.

${ }^{2}$ LTS5, École Polytechnique Fédérale de Lausanne (EPFL), Lausanne, Switzerland.

${ }^{3}$ Department of Radiology, University Hospital (CHUV), Lausanne, Switzerland.

${ }^{4}$ Centre d'Imagerie BioMedicale (CIBM), École Polytechnique Fédérale de Lausanne (EPFL), Lausanne, Switzerland.

${ }^{5}$ Department of Radiology, University of Geneva, Geneva, Switzerland.

${ }^{6}$ Siemens Medical Solutions USA, Inc., Boston, Massachusetts, USA.

*Correspondence to: Maryna Waszak, PhD, Siemens ACIT, EPFL QI-E, 1015 Lausanne, Switzerland. Tel: + 41 (0) 215459 974;

E-mail: maryna.waszak@gmail.com.

'These authors contributed equally to this work.

This work was supported by CIBM of the UNIL, UNIGE, HUG, CHUV, EPFL, and the Leenaards and Jeantet Foundations.

Received 19 April 2016; revised 19 June 2016; accepted 11 July 2016

DOI 10.1002/mrm.26364

Published online 16 August 2016 in Wiley Online Library (wileyonlinelibrary. com).
}

(C) 2016 International Society for Magnetic Resonance in Medicine.

\section{INTRODUCTION}

A better management of motion in MRI would be a great asset to clinical routine and research applications, and could render both clinical operation and research studies more cost effective as a result of increased patient throughput and to reduce the need for call-back exams. Despite many advances in the field of motion correction in MRI, no general solution has yet been found (1). All suggested correction techniques are limited by targeting specific sequences or to specific motion types like in-plane motion.

Motion correction methods are divided into two categories: prospective and retrospective. Prospective methods are applied during the sequence run time by periodically adapting the acquisition coordinate system to follow the imaged object, and have the potential both to compensate for considerably large motion amplitudes and to correct for spin history effects. These strategies traditionally involve either MR-based navigators of various complexity (2-5) external tracking systems like an optical camera with a marker (6) or active coils (7). External tracking hardware can provide very accurate motion information and be integrated with various sequences. However, such hardware relies on fiducial markers that might not be tolerated by the subject. Optical systems require an unobstructed field of view that can be impeded by radiofrequency (RF) coils or patient positioning aids. MR-based navigators are potentially valuable tools for prospective motion correction, as no additional hardware for external devices is required, but a tradeoff between tracking accuracy and navigator complexity must be considered. Higher navigator complexity provides better tracking and, hence, better correction results but might substantially prolong the acquisition time or might be incompatible with the target sequence design.

Retrospective methods, however, address the problem of motion after the image has been acquired and are, ideally, independent of the MR image acquisition sequence. A popular retrospective correction method is PROPELLER (8), in which the center of k-space is oversampled, to have redundant information that is subsequently used to adjust k-space lines for achieving better image quality. Another class of retrospective correction techniques is based on autofocusing (9-12). The advantage of these methods is that they can correct for nonrigid motion (13). However, they are limited by their inability to address large motion amplitudes, as they lead to unrecoverable missing k-space data.

In this work, we explore a prospective motion correction strategy using the recently proposed free-induction-decay 


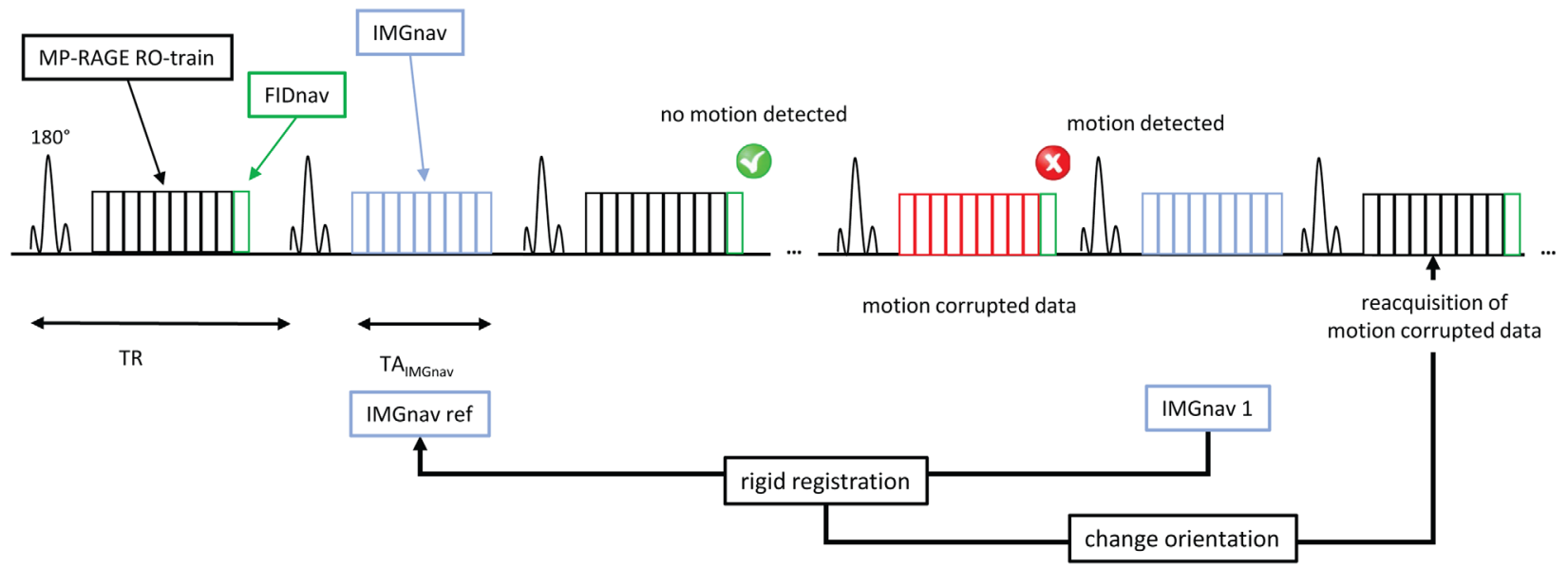

FIG. 1. Symbolic depiction of the proposed MPRAGE acquisition strategy with motion correction based on FIDnav-triggered IMGnavs (blue excitation train). The reference IMGnav is acquired in the beginning of the scan and then co-registered to an IMGnav, whose acquisition is driven by the FIDnav. The registration parameters are applied in the following TR to adjust the orientation for motion correction and reacquire the motion corrupted TR (red excitation train).

navigator (FIDnav) (14). The FIDnav refers to the measurement of a k-space center signal and was originally proposed to measure and correct for $\mathrm{B}_{0}$-field fluctuations $(15,16)$. The FIDnav was also proposed for shimming (17) or to monitor motion in abdominal imaging (18).

The FIDnav has been demonstrated to be sensitive to motion (14). In this work, it was employed for continuous $(v=0.43 \mathrm{~Hz})$ motion monitoring. Upon motion detection and only in this case, the acquisition of a more complex image navigator (IMGnav) is triggered for realtime motion correction. Both the FIDnav and IMGnav were implemented in a prototype magnetization-prepared rapid gradient-echo (MPRAGE) sequence and adapted to minimally affect the magnetization, acoustic noise level, and scan time of the acquisition. The proposed strategy presents an extension of other recently proposed approaches (2), by minimizing the scan time and/or gradient activity overhead arising from repetitive acquisition of an IMGnav.

\section{METHODS}

\section{Data Acquisition and Experimental Design}

The three-dimensional (3D) MPRAGE volumes were acquired with the following parameters: repetition time (TR)/inversion time (TI)/echo time (TE)/ $\alpha /$ time to acquisition $(\mathrm{TA})=2300 \mathrm{~ms} / 900 \mathrm{~ms} / 2.86 \mathrm{~ms} / 9^{\circ} / 5: 20$, echo spacing $=7 \mathrm{~ms}$, matrix $256 \times 256 \times 176,1.0 \mathrm{~mm}^{3}$ isotropic, $2 \times$ iPAT with 24 reference lines, Cartesian sampling scheme, closely resembling the updated ADNI protocol (19). The FIDnav acquisition module $\left(\alpha=9^{\circ}, 64\right.$ points in $0.2 \mathrm{~ms}, \mathrm{TI}=1532 \mathrm{~ms}$ ) and the IMGnav module (gradient echo (GRE), $\alpha / \mathrm{TA}=9^{\circ} / 1.2 \mathrm{~s}$, echo spacing $=7 \mathrm{~ms}$, six echoes, matrix size $64 \times 64 \times 32$, voxel size $4.1 \times 4.1 \times$ $6.0 \mathrm{~mm}^{3}$ ) were added to a prototype MPRAGE sequence. In this implementation, the FIDnav is acquired every TR following the gradient-echo block of the MPRAGE sequence. In contrast, the IMGnav is acquired only following the detection of a motion event (Fig. 1). The IMGnav is reconstructed and co-registered to a reference
IMGnav volume acquired in the beginning of the scan in real time. The sequence operates in two modes following the event of an IMGnav acquisition: (1) Motion parameters are computed and applied for prospective, quasi real-time correction of the acquisition coordinate system (MoCo-ON) and (2) motion parameters are computed but not used for prospective correction (MoCo-OFF). Motion correction was applied to all lines including the reference lines.

After obtaining written consent, five healthy volunteers were scanned with the prototype MPRAGE sequence at 3 Tesla (T) (Magnetom Verio, Siemens Healthcare $\mathrm{GmbH}$, Erlangen, Germany) operating under the VB17 software and using a 32-channel head coil. The respective experiments were labeled A.1-A.5 and refer to experiments in which subjects were asked to perform motion tasks during image acquisition either in mode MoCo-ON or MoCo-OFF (Table 1). Each subject also underwent the acquisition of two MPRAGE volumes in the MoCo-OFF mode after being instructed to stay still for the duration of those scans.

Additional five healthy subjects were scanned with a further variant of the prototype MPRAGE at $3 \mathrm{~T}$ (Magnetom Skyra, Siemens Healthcare GmbH, Erlangen, Germany) operating under the VD13 software and also equipped with a 32-channel head coil. The respective experiments were labeled B.1-B.5. The employed sequence variant acquired an IMGnav volume with each TR applying a motion trajectory from an external file (translations up to $17.9 \mathrm{~mm}$ and rotations up to $5.5^{\circ}$ ) while the subjects were asked to stay motionless. The employed motion trajectory (head nodding, shaking, and translation along the head-feet direction) had been recorded from a moving subject by an optical motion tracking system (20) in an earlier study (21). An update of the acquisition coordinate system was performed in real time with every TR. In other words, the time series of IMGnav volumes exhibited motion as "injected" by the external motion trajectory similar to the work from (22). The successively acquired IMGnavs were registered 
Table 1

Data Acquisition Setting with Respective Motion Pattern.

\begin{tabular}{lccc}
\hline Data set & $\begin{array}{c}\text { Motion correction } \\
(\text { MoCo) setting }\end{array}$ & Motion pattern & $\begin{array}{c}\text { Scanner type and } \\
\text { software version }\end{array}$ \\
\hline A.1-A.5 & MoCo-ON & $\begin{array}{c}\text { nodding motion, shaking motion, } \\
\text { z-translation } \\
\text { nodding motion, shaking motion, } \\
z \text {-translation, no motion } \times 2\end{array}$ & Verio, 3T, VB17 \\
B.1-B.5 & MoCo-OFF & 100xIMGnav with motion injection & Skyra, 3T, VD13 \\
\hline
\end{tabular}

to the reference IMGnav to experimentally obtain the motion parameters for comparison with the known input motion trajectory.

\section{Motion Paradigms during In Vivo Scans with Voluntary Motion}

The subjects scanned in the experiments A.1-A.5 were instructed to change their head position three times during a scan upon verbal commands and to follow frequently observed motion patterns (23): translation in head-feet direction, head nodding, and head shaking. The motion was executed as a one-time event by repositioning the head accordingly and remaining in this position until the next verbal command. During the scan, one of the patterns was performed three times upon verbal request (at the acquisition of the k-space line numbers 65, 128, and 191). These scans were repeated by having the motion correction turned on (mode MoCo$\mathrm{ON}$ ) and off (mode MoCo-OFF), resulting in six volumes per subject. Two additional data sets (I, II) for scan/ rescan comparison without voluntary motion in mode MoCo-OFF were acquired for each subject, resulting in a total of 40 MPRAGE volumes in this study (Table 1).

In both MoCo-ON and MoCo-OFF modes, the detection of motion by the FIDnav module results in the acquisition of an additional IMGnav and the computation of the six motion parameters (rigid body model). However, only in the MoCo-ON mode is the prospective correction of the acquisition coordinate system applied. In those acquisitions, to assess the effect of reacquisition, two sets of images were reconstructed, namely, the first set using the reacquired repetition after a motion event and the second set using the motion-corrupted data (Fig. 1).

\section{FIDnav Acquisition and Calculation}

The FIDnav is evaluated in real time to monitor and to detect motion events based on threshold criteria. The following two FIDnav threshold criteria were employed for all subjects and scans: (a) a threshold denoted as FID slow and (b) a threshold denoted as FID fast .

The FID slow was introduced to detect slow motion (eg, muscle relaxation) and $F I D_{\text {nav }}^{\text {fast }}$ to detect fast head motion (eg, rapid head turning). Overall, this approach was considered to be more sensitive to motion than the method proposed in (14), as only channels with maximum signal change were used. Empirically derived thresholds of 5\% change in FID signal for slow movements and $8 \%$ for fast movements were employed. As learned from previous acquisitions, a threshold of $6 \%$ for $F I D_{\text {nav }}^{\text {fast }}$ as used in a previous study (24) proved to be too stringent with respect to non-motion-related FID signal fluctuations (increased number of false-positive events).

For the detection of head movements using $F I D_{\text {nav }}^{\text {fast }}$, a scalar FIDnav value was calculated by combining the signals from the three coil elements that exhibited maximal signal changes among all 32 receive channels. We used the average of three coils to minimize the effect of random signal fluctuations, and at the same time maximizing the motion-induced signal change. Rapid motion at a time point $t$ was detected by analyzing signal differences from the preceding repetition at $(t-1)$, whereas slow movements at time $t$ were reflected by FIDnav differences from the first repetition of the scan (see Eq. [1]). Here, $|s(t)|$ is the absolute value of the complex average of all points from one single FID readout acquired in the th repetition in a coil element $n$. The avg $\max _{3 C}$ operator takes the FIDnav signal from those three coil elements in which the signal change is maximal and averages them. For the calculation of the $F I D_{\text {nav }}^{\text {slow }}$, the $\operatorname{avg}_{\text {AllC }}$ operator was used, which takes the average over all coil elements. The first two signal time points (equaling the first two TRs) were excluded from the FIDnav calculation for both $F I D_{\text {nav }}^{\text {fast }}$ and $F I D_{\text {nav }}^{\text {slow }}$ as a result of the signal not being in steady state yet, and the subsequent third signal time point was used as reference. Hence, the motion detection started at the fourth TR. If either FID fast or FID $D_{\text {nav }}^{\text {slow }}$ exceeded the empirical threshold values, then the respective TR was considered to contain a motion event.

$$
\begin{gathered}
F I D_{\text {nav }}^{\text {fast }}(t)=\operatorname{avg} \max _{3 C}\left|\frac{|\bar{s}(t)|-|\bar{s}(t-1)|}{|\bar{s}(t-1)|}\right| \\
F I D_{\text {nav }}^{\text {slow }}(t)=\underset{\text { AllC }}{\operatorname{avg}}\left|\frac{|\bar{s}(t)|-|\bar{s}(3)|}{|\bar{s}(3)|}\right|
\end{gathered}
$$

\section{Image Navigator Acquisition}

For the IMGnav, we used a recently proposed multiecho segmented "hybrid" 3D GRE prototype sequence (25) that was modified to employ a cylindrical k-space sampling pattern as shown in Figure 2b. The standard MPRAGE acquisition scheme was adapted to acquire multiple echoes using bipolar readout gradients. Phaseencoding gradients were inserted between the readout gradients to sample multiple portions of k-space after each excitation pulse. The reordering scheme was modified to group first echoes in the center of k-space to ensure the intended contrast (Fig. 2a). A phase 

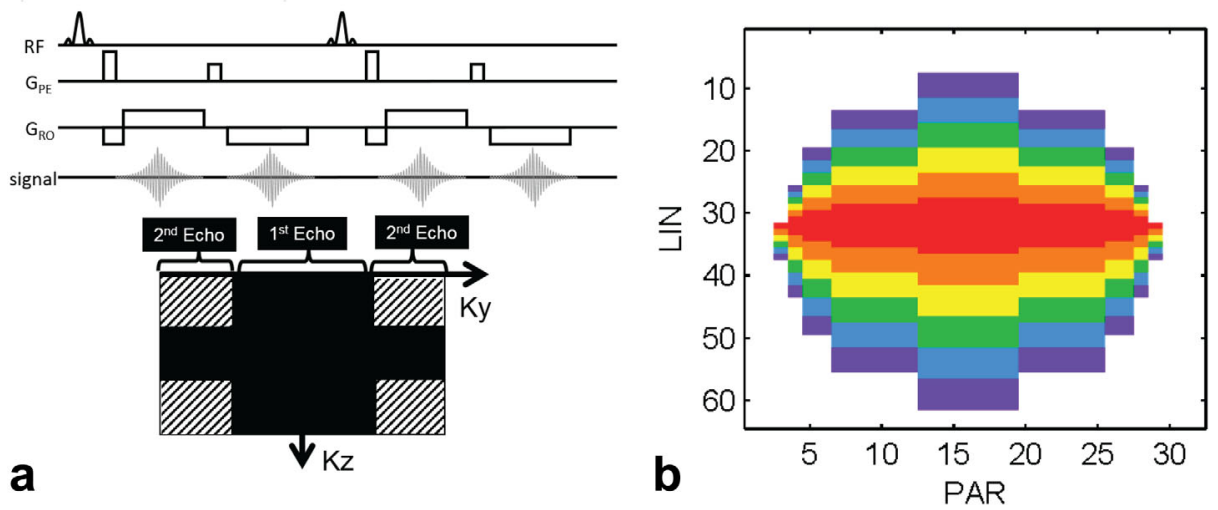

FIG. 2. (a) Acquisition sequence of the IMGnav in an example with two echoes. (b) Acquisition design of the IMGnav; different colors label the echo number (red, first echo; orange, second echo; yellow, third echo; green, fourth echo; blue, fifth echo; purple, sixth echo), whereas in white the nonacquired k-space locations are labeled and are filled with zeroes for reconstruction.

correction algorithm similar to the one used for echoplanar imaging (EPI) acquisitions was applied to remove phase inconsistencies between k-space lines acquired in the echoes with differing polarity, in which the correction was based on the lines acquired in the center of kspace.

The IMGnav protocol $\left(\alpha / \mathrm{TA}=9^{\circ} / 1.2 \mathrm{~s}\right.$, echo spacing 7 ms, six echoes, matrix size $64 \times 64 \times 32$, voxel size $4.1 \times 4.1 \times 6.0 \mathrm{~mm}^{3}$ ) was designed specifically to acquire a whole-brain volume with very similar properties as the GRE imaging block of the host MPRAGE. Particular attention was paid to maintain acoustic noise and longitudinal magnetization as similar as possible and not to disturb it by the acquisition of the IMGnav. The nonacquired k-space points outside the cylindrical sampling pattern were zero-filled for image reconstruction (Fig. 2b).

\section{Quantitative Image Quality Assessment Based on Automated Brain Volumetric Analysis and Image Quality Index}

Motivated by recent studies $(26,27)$, the performance of the proposed correction method was also assessed by conducting brain tissue segmentation using an automated morphometry package (28). The segmentation results of the scans with (a) motion and correction (MoCo-ON), (b) motion but without correction (MoCo-OFF), and (c) without motion and without correction, were compared. We calculated the normalized volumetric differences $\Delta$ between the "no motion" $V_{\text {nomotion }}$ and "motion" $V_{\text {motion }}$ cases for total intracranial volume $\left(\Delta_{\text {TIV }}\right)$, global white matter $\left(\Delta_{\mathrm{WM}}\right)$, and gray matter $\left(\Delta_{\mathrm{GM}}\right)$ :

$$
\Delta=\left|\frac{V_{\text {motion }}-V_{\text {nomotion }}}{V_{\text {motion }}+V_{\text {nomotion }}}\right|
$$

Per each subject, $\Delta$ is a measure of the change in volume between two acquisitions normalized by their sum. It is a dimensionless quantity. Its values are in the interval $[0,+1]$ and represent a normalization of the absolute volume variance in the absence of a ground truth volume, to be able to compare the results across subjects.
Finally, a quantitative image-quality assessment based on the background noise evaluation as described in $(29,30)$ was performed on all MPRAGE volumes to assess the change in the quality index (QI) with and without correction, and without reacquisition.

\section{RESULTS}

\section{Registration Accuracy}

Figure 3a shows an IMGnav from one subject. Ghosting artifacts in the anterior-posterior direction (phase encoding) on the sagittal and axial views are visible. These artifacts are present in all IMGnav acquisitions. In Supporting Figure S1a, the external motion trajectory is depicted, which introduces translations up to $17.9 \mathrm{~mm}$ and rotations up to $5.5^{\circ}$ in the experiments B.1-B.5 to assess the registration accuracy.

These validation experiments revealed that the employed registration framework was able to quantify the six rigid-body motion parameters with a mean absolute error of $0.27 \pm 0.38 \mathrm{~mm}$ and $0.19 \pm 0.24^{\circ}$. The amplitude of translational and rotational registration errors was found to be higher with increasing motion amplitudes of the prescribed motion trajectory (Supporting Fig. S1b). However, with the exception of very small motion amplitudes (translation $<0.01 \mathrm{~mm}$ and rotation $<0.05^{\circ}$ ), relative errors in this study were found to be below $10 \%$ of the respective motion amplitude. We observed maximal registration errors of up to $2.0 \mathrm{~mm}$ and $1.3^{\circ}$ (Fig. 3b).

\section{Sensitivity of Motion Detection and Motion Correction}

In the experiments A.1-A.5, verbal commands were given to the subjects to execute the described motion tasks. We detected motion amplitudes of up to $11 \mathrm{~mm}$ translation and $9^{\circ}$ rotation (Supporting Table S1). A substantial fraction of detected and corrected motion events relate to the execution of motion tasks; however, more motion events were detected by the FIDnav. For example, during scans in which the subject was asked to remain still, triggering events occasionally occurred, indicating the 

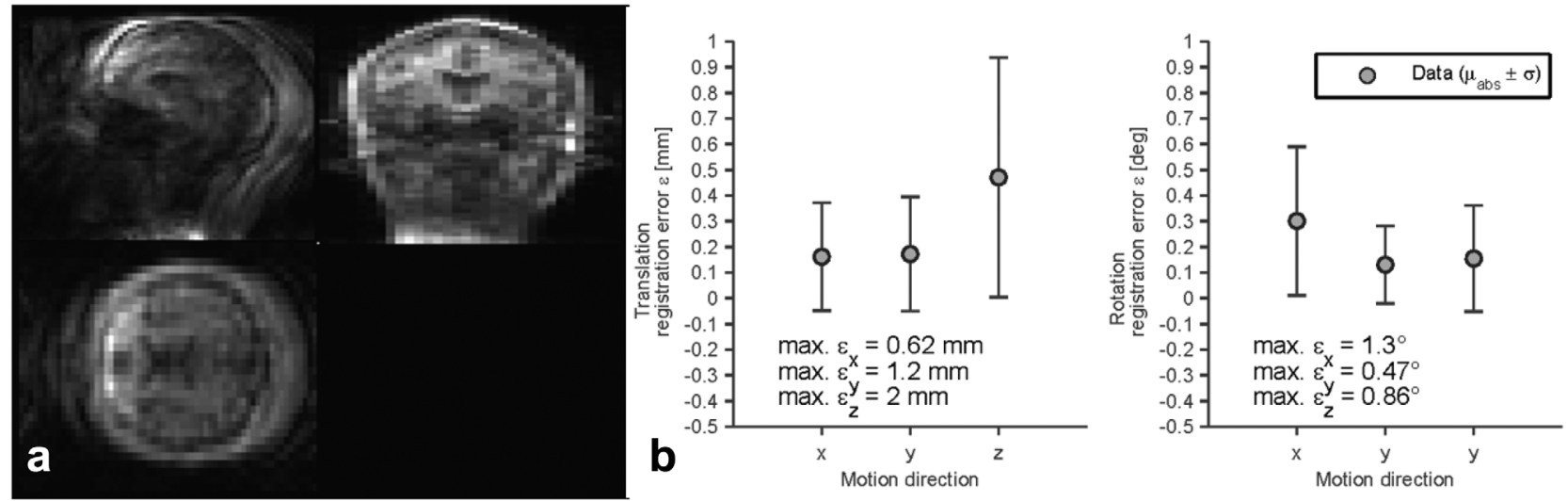

FIG. 3. (a) Sagittal, coronal, and axial views of the IMGnav. (b) Registration error for translations and rotations for all five subjects with its mean of the absolute error and standard deviation. The maxima of the detected registration errors are $0.62,1.2$, and $2 \mathrm{~mm}$ for $x, y$, and $z$ translation, and $1.3^{\circ}, 0.47^{\circ}$, and $0.86^{\circ}$ for rotations around $x, y$, and $z$, respectively.

sensitivity to motion and/or potential false-positive detections. Specifically, the acquisition of the IMGnav was in three cases initiated by the $F I D_{\text {nav }}^{\text {slow }}$ approximately after two-thirds of the total scan time, as shown in Figure 4 . Here, the registration parameters indicated very small positional changes of the head in the range of $1 \mathrm{~mm}$ and $1^{\circ}$.

In the case of subject A.1, a high number of triggering events were detected in "no motion" scans, as shown in Figure 5a (16 and 23 triggering events for the two scans without subject motion). The reported registration parameters did not indicate any substantial positional changes of the head, and the image did not show any obvious motion artifacts. Therefore, these triggering events are considered as false-positive events, although a true but irrelevant object movement may have caused the detection (eg, deep breathing without head movement in the frame of reference).

True motion events (after a verbal command to change the head position) were detected in all cases, and the acquisition of the IMGnav was reliably triggered to provide the new positional parameters to the sequence and
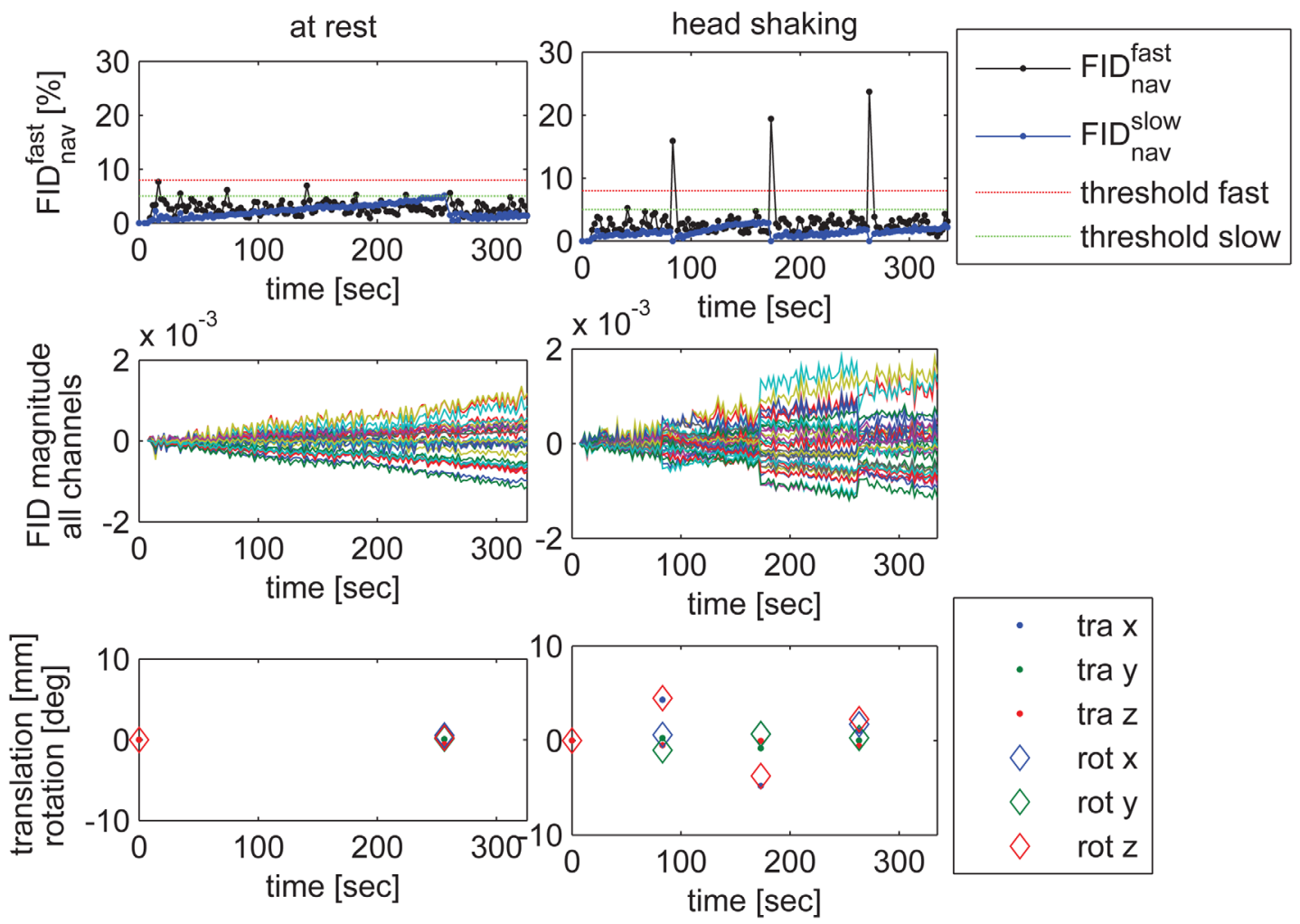

FIG. 4. Triggering events and the corresponding motion trajectories, in addition to the normalized FID signal magnitude from all 32 coil elements. The shown FID signal was shifted to zero for better visual representation. FID nav and FID nast for a subject at rest (left) and performing shaking motion (right). 

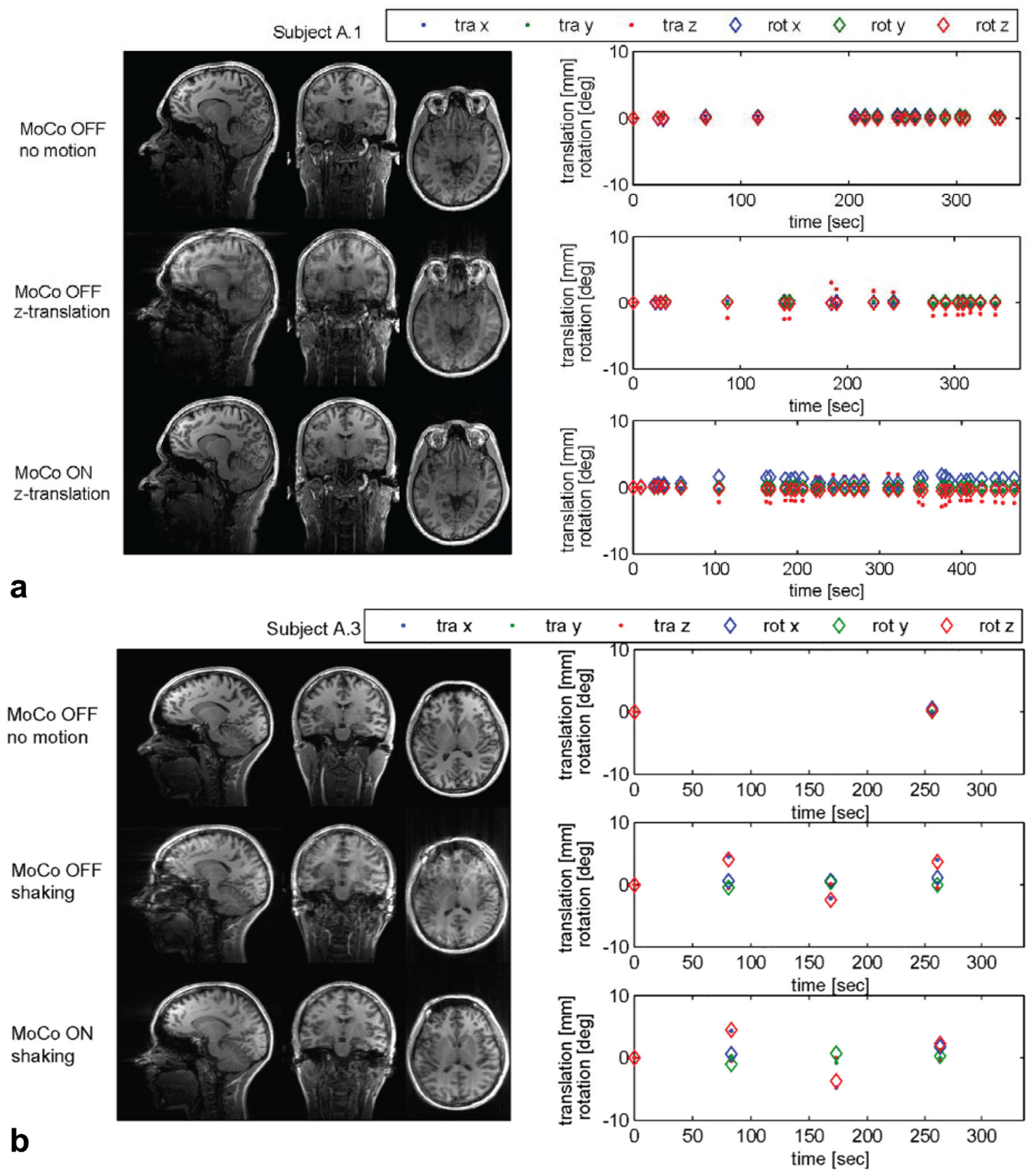

FIG. 5. Motion correction results from the data set A together with the detected motion trajectory for different subjects and motion patterns. An image acquired without any deliberate subject motion is shown for reference compared with the images with and without the motion correction mechanism in place. Here, the $F I D_{\text {nav }}^{\text {slow }}$ and $F I D_{\text {nav }}^{\text {fast }}$ were used for the detection of motion events: (a) subject A.1 with z-translation motion pattern; (b) subject A.3 with shaking motion pattern.

to reacquire the motion-corrupted repetition. In some cases, several triggering events were detected after a verbal command was given to the subject to change his or her head position. In these cases, the registration parameters showed that indeed the subject could not hold the new position and moved his or her head even further or slightly back toward the original position (Supporting Fig. S2a, MoCo-OFF, z-translation). This behavior was also verbally confirmed by the subject after the experiment.
In the experiments in which subjects received verbal instructions, three motion events per volume were expected. In this investigation, we observed in total 90 true positive and 235 false positive motion events and zero false negatives events. Each MPRAGE acquisition consisted of 139 repetitions plus the additionally reacquired repetitions. Therefore, out of 5560 total repetitions in 40 acquired volumes (from subjects A.1-A.5) with 5440 possible triggering events, we report a sensitivity of $100 \%$ and a specificity of $96 \%$. 

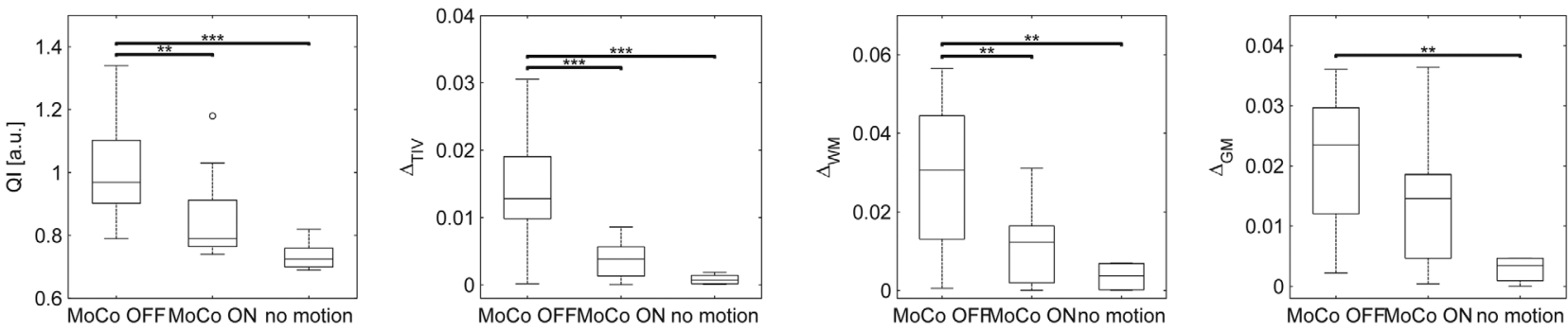

FIG. 6. Quantitative evaluation of the image quality metric $\mathrm{QI}$ and the morphological brain volume segmentation results for the data acquired without (MoCo-OFF) and with motion correction (MoCo-ON) compared with data acquired without motion (no motion) and without motion correction. Data from all subjects and experiments are shown. The stars indicate the significance level $P$ of a rank-sum test (no star represents $\mathrm{P}>0.05$, * represents $P \leq 0.05$, ${ }^{\star \star}$ represents $P \leq 10^{-2}$, ${ }^{\star \star \star}$ represents $P \leq 10^{-3}$ ).

Each motion detection event prolongs the scan time by two TRs with one TR (2300 ms) for the acquisition of the IMGnav and one TR for the reacquisition of the motioncorrupted repetition. On average, 8.1 motion events per volume scan were detected (see Supporting Table S1) during the acquisition of the 40 volumes. This includes both true and false-positive events. The additional average time being spent as a result of the enabled motion correction was $39.7 \mathrm{~s}$ per volume in motion-corrupted scans. This corresponds to a scan time prolongation of approximately $10 \%$ for an MPRAGE protocol. This accounts for $2.3 \mathrm{~s}$ for the reference IMGnav $+(2.3 \mathrm{~s}$ for the triggered IMGnav +2.3 for reacquisition)*8.1 motion events on average. In the scans when the subject was asked to stay still, an average of 4.9 motion events were detected by the FIDnav, which corresponds to an average of 24.8 s extra scan time per scan.

As shown in Figure 5 and Supporting Figure S2, artifacts are visible in images without motion correction. The visual improvement of motion-corrected compared with uncorrected images is apparent. However, not all motion artifacts could be removed. In the motioncorrected images, residual ringing artifacts, blurring, or ghosting was visible (Fig. 5b).

\section{Quantitative Assessment of Image Quality Improvements after Motion Correction}

Quantitative evaluation comparing the intrasubject reproducibility of segmentation results based on the scan/ rescan data (no-motion experiments, MoCo OFF) showed $\Delta_{\mathrm{TIV}}=1 \times 10^{-3}, \Delta_{\mathrm{WM}}=4 \times 10^{-3}$, and $\Delta_{\mathrm{GM}}=3 \times 10^{-3}$ with a standard deviation of $1 \times 10^{-3}$ for TIV, $3 \times 10^{-3}$ for white matter, and $2 \times 10^{-3}$ for gray matter. This is in line with the observations of the intrasubject variability as reported previously (31).

By applying the proposed motion correction methodology, motion-induced volumetric measurement errors could be significantly reduced in our experiments from $14 \times 10^{-3}$ to $4 \times 10^{-3}$ for $\Delta_{\text {TIV }}$ and from $30 \times 10^{-3}$ to $11 \times 10^{-3}$ for $\Delta_{\mathrm{WM}}$ (wrt being the volumes acquired without motion) (Fig. 6). The motion-induced error for gray matter $\Delta_{\mathrm{GM}}$ was reduced from $21 \times 10^{-3}$ to $14 \times 10^{-3}$, but not significantly $(P=0.09)$.

Overall, the quantitative image quality results confirmed that our motion correction improved the image quality. As shown on Figure 6, QI could be significantly reduced from 1.01 to 0.86 (the lower the QI value, the higher the image quality). However, our correction could not bring the image quality up to the "no motion" ultimate target (mean QI being 0.74), even though the difference is not significant. Similarly, our correction could not reduce motion-induced volumetric errors down to the no-motion scan/rescan target.

\section{Effect of Reacquisition on Image Quality}

We also investigated whether the reacquisition of presumably motion-corrupted repetitions adds value to the images in terms of image quality and segmentation results. The effect of motion on image quality depends heavily on the location in k-space where it occurred. In our experiments, the motion-corrupted lines were in three locations (ie, after line numbers 65, 128, and 191). To that end, two different reconstructions were compared, in which the reacquired k-space lines were either used or discarded. In line with previous publications (2), we observed in the reconstructions with reacquisition less ringing in the brain region and background for all inspected motion patterns (Fig. 7). This observation was also confirmed by the quantitative evaluation of the quality index. As shown in Figure 8, a mean QI of 1.07 was observed for images in which no reacquisition was applied that is not significantly different from the QI of images without any motion correction (QI without correction: 1.01). The trend for slightly improved QI with reacquisition can be explained by QI's sensitivity to image background artifacts. However, the quantitative volumetric assessment of tissue structures revealed no evident differences in the brain volume segmentation between motion-corrected images with and without reacquisition (Fig. 8).

\section{DISCUSSION}

\section{Registration Accuracy of IMGnav}

We assessed the registration accuracy of the IMGnav by externally changing the acquisitions coordinate system during scanning (ie, "injecting" artificial motion). This provides a ground-truth motion trajectory for the coregistration performance assessment. It should be noted that true unintentional positional head changes during the scan may also lead to degraded shim performance, and may change or introduce additional artifacts such as 


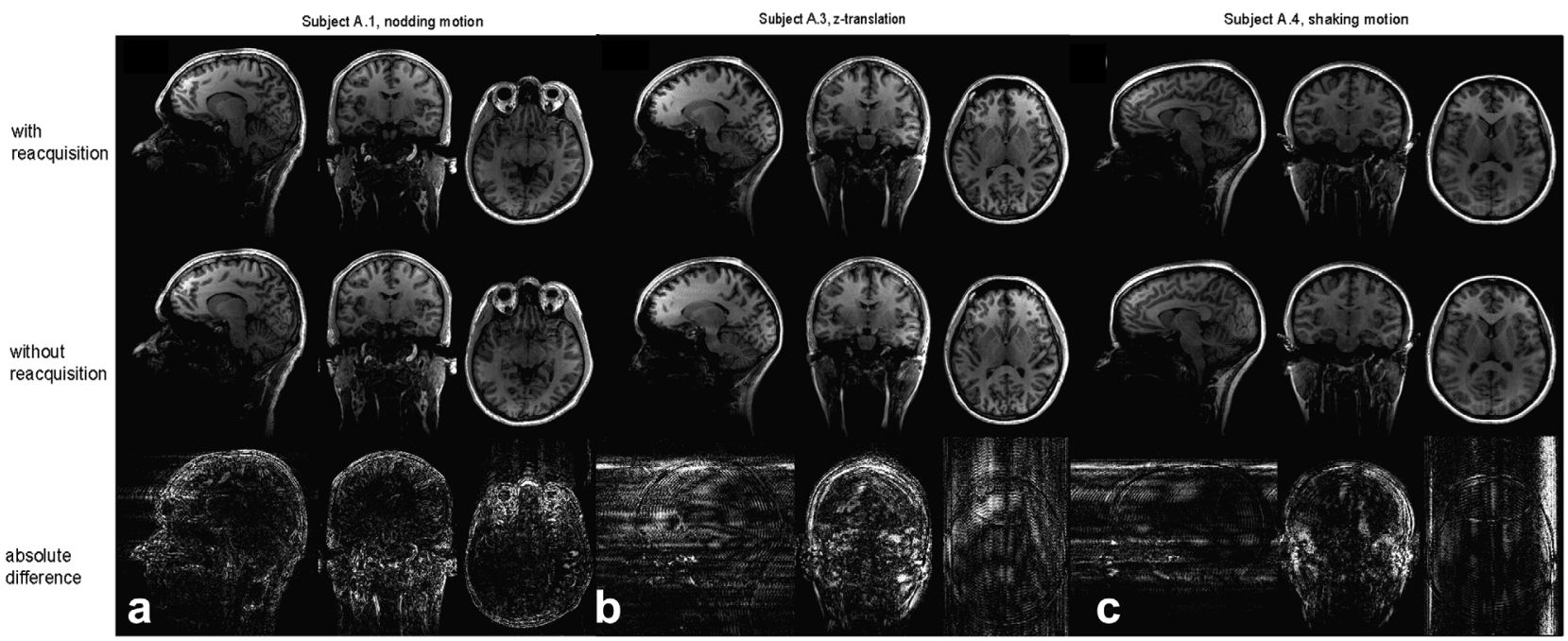

FIG. 7. Reconstruction results from data with and without using the reacquired repetitions, and the absolute difference image from the two different reconstructions. (a) Subject A.1 performing nodding motion. (b) Subject A.3 performing motion in head-feet direction (ie, z-translation). (c) Subject A.4 performing shaking motion.

geometric distortions or even motion artifacts within the IMGnav image volume, potentially leading to reduced registration accuracy. In contrast, we assume that small motion amplitudes with translation amplitudes below $10 \mathrm{~mm}$ and rotations below $8^{\circ}$, which holds true for$>90 \%$ of motion events in experiments A.1-A.5, have no or subtle effects on shim status and image artifacts as proposed in (5). Another reason for elevated registration errors could be the hyperintensities in the IMGnav caused by the bias field from the coil sensitivity profile of the 32-channel coil, as no bias field correction was used in the image reconstruction of the IMGnav.

A possible way to assess the motion-estimation capabilities of the proposed method would be the use of a physically moving object, eg, a moving phantom, in which the ground truth motion coordinates are exactly known. Unfortunately, it is not trivial to construct such a device that exhibits similar MRI properties as a human head in terms of susceptibilities, relaxation properties, and loading. Another possibility to assess the registration accuracy of the IMGnav could be the use of an additional independent motion-tracking technique as, for example, an optical camera system to provide the gold-standard motion parameters of a moving human subject as performed in (32). Alternatively, another image navigator with known high registration accuracy as, for example, the one proposed in (33) could be used for cross validation of the registration performance of our IMGnav.

The proposed IMGnav exhibits remaining ghosting artifacts in the anterior-posterior phase-encoding direction. One of the sources of these artifacts can be the inconsistency in signals of different echoes that arises from hyperintense signals that come from fat in the neck region. We did not observe any signs that these ghosting artifacts affected the registration accuracy in this study, but see the potential to improve the image navigator acquisition and performance, which can only lead to an improved robustness of the co-registration performance.

Several motion correction techniques using an imaging navigator have already been proposed. For each of the methods, the motion prediction performance of the navigator was also assessed. The registration accuracy of the
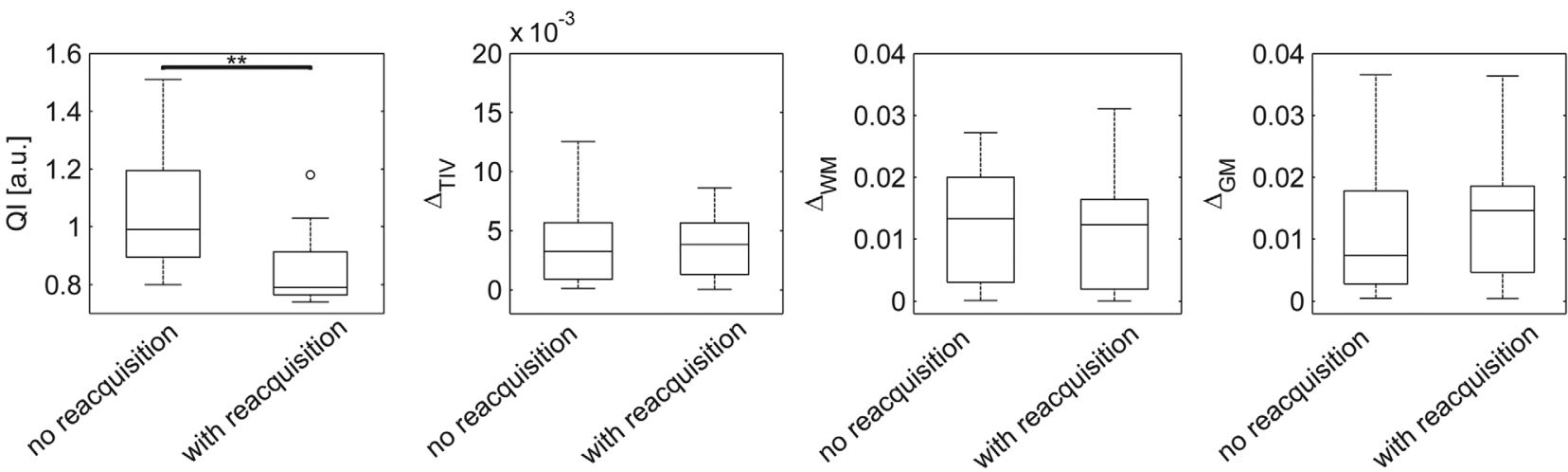

FIG. 8. Quantitative evaluation of the image quality metric QI and the morphological brain volume segmentation results for data acquired with motion correction on but reconstructed with and without the use of the reacquired repetitions. Data from all subjects from set $A$ are shown. The stars indicate the significance level $P$ of a rank-sum test (no star represents $P>0.05,{ }^{*}$ represents $P \leq 0.05$, ${ }^{\star *}$ represents $P \leq 10^{-2}$, ${ }^{\star * *}$ represents $P \leq 10^{-3}$ ). 
vNAV (volumetric navigator) (2) is reported to be in the micrometer and $10^{-5}$ degree range. Another study showed that the motion detected by the vNAV from a subject at rest was below $1 \mathrm{~mm}$ and $1^{\circ}$ (33) and did not influence the sequence targeted for correction. PROMO (4) reports motion prediction errors below $10 \%$ of applied ground truth motion, and tNAV (through-plane navigator) is able to provide motion parameters with an accuracy of $\pm 0.5 \mathrm{~mm}$ and $\pm 0.5^{\circ}$ (34). In a study in which the motion detection accuracy of PROMO was compared with a camera system for motion tracking (32), the authors report a registration accuracy with a mean absolute error within $1 \mathrm{~mm}$ and $1^{\circ}$ with maximal errors up to approximately $2.2 \mathrm{~mm}$ and $2.9^{\circ}$. Summarizing, we conclude that the reported accuracy (a) reflects closely the performance in the real-world experiments and (b) provides-congruent with reviewed literature-the best-case accuracy without taking into account the possible unintentional movements of the subject during the artificial introduction of the motion trajectory. Although the accuracy evaluation of the IMGnav proposed in this work showed similar results (mean absolute registration error of $0.27 \pm 0.38 \mathrm{~mm}$ and $0.19 \pm 0.24^{\circ}$ and maximal errors of up to $2.0 \mathrm{~mm}$ and $1.3^{\circ}$ ), we see potential for improvement to further increase the image quality of corrected data.

\section{FIDnav-Triggered Motion Detection and Correction}

An FID navigator can be added to practically any sequence, in many without having an effect on scan time or the magnetization profile of the host sequence. The FI $D_{\text {nav }}^{\text {slow }}$ and FID fast allow the detection of motion events reliably after reaching steady state (after two repetitions in the case of MPRAGE). However, the main challenge is defining the triggering threshold. Our choice of the threshold was based on empirical values ( $F I D_{\text {nav }}^{\text {slow }}, F I$ $D_{\text {nav }}^{\text {fast }}$ ), as an automated thresholding mechanism was not within the scope of this implementation and is considered future research at this step. Ideally, different threshold values for motion detection would apply for different subjects; it was shown that the mapping from FIDnav signal changes to the underlying motion trajectory is subject-specific (21).

The crux of setting an optimal triggering threshold is the missing knowledge about the natural FIDnav signal fluctuation for the specific subject and scan. This information, however, could be acquired during the sequence adjustment and preparation phase. Similarly, the sequence could be started with a "training phase," ie, a set of IMGnavs acquired in parallel to FIDnavs that are then used to assess the possible presence of motion. By registering the IMGnav, it would be possible to assess the motion parameters during the training phase. If no motion was present during the acquisition of the IMGnav set, it would then allow setting the triggering threshold value to be above the natural variance of the FIDnav signal individually for the specific subject during the scanning session to follow. If motion was present during this training phase, then the resulting motion parameters could be used to find the linear combination of the multichannel FIDs to predict the motion during the image acquisition without the further need of the IMGnav but based only on the FID signal as shown in (21). Such an approach would even benefit from a wide range of motion.

We report additional scan time that was spent in our experimental setup as a result of enabled motion correction. These numbers, however, might not comprehensively represent realistic clinical scenarios. Furthermore, our experiments did not cover other motion patterns such as continuous motion or nonrigid motion. Nonrigid motion, like swallowing, influences the FIDnav (35) and might cause false-positive triggering events. It would be possible to detect slow, continuous motion by the $F I D_{\text {nav }}^{\text {slow }}$, as well as FID signal drifts, as observed in (21), causing the $F I D_{\text {nav }}^{\text {slow }}$ to detect a false-positive motion event. We observed this to be a rare scenario, and the benefit of the ability to detect slow motion justifies its use. Furthermore, it is known that the FIDnav signal is also sensitive to $\mathrm{B}_{0}$ field fluctuation as already exploited in $(15,16)$; this could further contribute to false-positive triggering events if such field changes are not motion related.

\section{Advantages and Limitations of the Proposed Motion Correction Method}

The present approach extends recent work on MR-based motion correction (2) in the sense that motion is monitored with practically no time penalty based on fast and low-impact FIDnavs, and the imaging navigators are employed only when needed without influencing the magnetization profile of the host sequence. We propose the IMGnav, which is designed specifically to match the properties of the host MPRAGE sequence, in terms of acoustic noise and magnetization profile (ie, the same number of excitations during a readout train). The sound during the acquisition of the IMGnav differs slightly from the MPRAGE readout, which is less sudden than an EPI volume and could be considered beneficial as a biofeedback to the subject that a motion event was detected and the subject has to remain still.

It could be shown that significant improvement in image quality from scans with deliberate subject motion can be achieved when employing the proposed method. To benchmark the motion correction performance, we chose the segmentation results and a quality index based on image background evaluations. The segmentation discrepancies could be reduced by the proposed motion correction for the total intracranial volume and for white matter. The same trend is observed for gray matter without those results being statistically significant $(P=0.09)$.

Although the motion-related artifacts could be reduced, the quality of the "no motion" images could not be achieved in all cases. Residual ringing and ghosting artifacts were visible in images with motion correction. We assume that this is caused by the limited registration accuracy of the IMGnav and k-space inconsistencies. However, the reported values are in line with other motion correction methods using similar technology (vNAV, PROMO).

Noteworthy, the settings of the IMGnav would have to be adapted with respective changes in the protocol, as 
the main design decision for the IMGnav was to employ the same or a similar number of excitations as the MPRAGE host sequence to avoid strong changes in the acoustic pattern of the host sequence as a result of the navigator.

In the current proof-of-concept implementation, we did not set any limit to the reacquisition of the presumably corrupted echo trains. Theoretically, if the subject continuously moves, there would be an infinite number of reacquisitions and the sequence would never stop. In (2), the authors propose to move the reacquisition to the end of the scan and limit it to a preset number of echo trains with most severe motion. Another alternative might be to reacquire the corrupted repetitions immediately, but limit it to a single reacquisition per repetition to ensure that the sequence would eventually end. The simplest and most efficient solution would be not to reacquire any repetitions at all. Our evaluations showed that reacquisition, although providing visually more appealing images, does not add significant value in terms of volume segmentation discrepancies in our investigation or if motion parameters are in the submillimeter range and appear in the high-frequency area of $\mathrm{k}$ space. More importantly, such remaining artifacts may be removed by complementing the prospective correction by a retrospective FID-guided (36) or blind (10) correction. Nevertheless, the current study demonstrates that such a concept, which used the FID signal, can provide valuable improvement in image quality when motion is present during the acquisition.

A growing interest in integrated positron emission tomography (PET)/MRI provides another venue for the need of motion correction techniques (37). MR-based motion correction offers an efficient way to correct for motion-induced artifacts in the MR image and during PET image reconstruction $(38,39)$. Here, we would like to point out that our method allows for motion monitoring with the help of a simple MR signal, the FIDnav, and for motion correction when needed by incorporating an MR-based IMGnav. The motion parameters as extracted from the registration of the IMGnav could be also used to mitigate motion-related artifacts in integrated PET/MRI.

\section{Future Work}

Future work should aim at the implementation of a subject-specific FIDnav threshold criteria to reduce the scan time overhead resulting from false-positive detection of motion events and possibly enabling a IMGnav acquisition through a motion prediction approach $(21,40)$. In addition, the implementation of an efficient reacquisition strategy would refine the motion correction capability of the proposed method. Additional experiments are needed to reveal the reason for residual motion artifacts (ie, ringing and ghosting) in the image despite active motion correction, as our experiments could show a registration accuracy of the IMGnav to be comparable with other motion correction methods that use similar technology and do not report related remaining degradations of image quality. It would also be of interest to investigate ways of mitigating the ghosting artifact in the IMGnav, to possibly further increase the registration accuracy. Moreover, it is of interest to define the limits for correctable motion magnitudes similar to Prospective Acquisition CorrEction technique (PACE) (5). It would be also interesting to extend the proposed motion correction strategy, employing FIDnav-driven triggering of IMGnavs to other sequences including spin-echo and basic gradient echo sequences. Twodimensional imaging might be of interest here especially (eg, turbo-spin-echo sequence). An experiment dedicated to slow motion, such as caused by muscle relaxation, would be valuable to investigate more realistic motion scenarios.

\section{CONCLUSIONS}

We applied FID navigators to efficiently monitor head motion in a prototype MPRAGE sequence as already proposed in (14), and extended the methodology by a prospective motion correction mechanism through ondemand, activated image navigators. The image navigators were specifically designed to match the acoustic noise and the magnetization profile of the host MPRAGE sequence. We investigated the motion tracking capability of the proposed image navigator in human scans showing submillimeter and subdegree mean accuracy and precision. Significant, qualitative image quality improvement was evident in experiments with moving human volunteers with the proposed motion correction methodology.

\section{ACKNOWLEDGMENTS}

The authors wish to thank Sebastian M. Waszak, Alexis Roche, Davide Piccini, Mario Fartaria De Oliveira, Dylan Tisdall, Andre van der Kouwe, and David Faul for valuable discussions and suggestions.

\section{REFERENCES}

1. Zaitsev M, Maclaren J, Herbst M. Motion artifacts in MRI: a complex problem with many partial solutions. J Magn Reson Imaging 2015;42: 887-901.

2. Tisdall D, AT, Reuter M, Meintjes EM, Fischl B, van der Kouwe AJW. Volumetric navigators for prospective motion correction and selective reacquisition in neuroanatomical MRI. Magn Reson Med 2012;68: 389-399.

3. van der Kouwe AJW, Benner T, Dale AM. Real-time rigid body motion correction and shimming using cloverleaf navigators. Magn Reson Med 2006;56:1019-1032.

4. White N, Roddey C, Shankaranarayanan A. PROMO: real-time prospective motion correction in MRI using image-based tracking. Magn Reson Med 2010;63:91-105.

5. Thesen S, Heid O, Mueller M, Schad LR. Prospective acquisition correction for head motion with image-based tracking for real-time fMRI. Magn Reson Med 2000;44:457-465.

6. Zaitsev M, Dold C, Sakas G, Hennig, J, Speck O. Magnetic resonance imaging of freely moving objects: prospective real-time motion correction using an external optical motion tracking system. NeuroImage 2006;31:1038-1050.

7. Ooi MB, Aksoy M, Maclaren J, Watkins RD, Bammer R. Prospective motion correction using inductively coupled wireless RF coils. Magn Reson Med 2013;70:639-647.

8. Pipe JG. Motion correction with PROPELLER MRI: application to head motion and free-breathing cardiac imaging. Magn Reson Med 1999;42963-969.

9. Atkinson D, Hill DLG, Stoyle PN, Summers PE, Keevil SF. Automatic correction of motion artifacts in magnetic resonance images using an entropy focus criterion. IEEE Trans Med Imaging 1997;16:903-910. 
10. Loktyushin A, Nickisch H, Pohmann R, Schölkopf B. Blind retrospective motion correction of MR images. Magn Reson Med 2013;70: 1608-1618.

11. McGee KP, Manduca A, Felmlee JP, Riederer SJ, Ehman RL. Image metric-based correction (autocorrection) of motion effects: analysis of image metrics. J Magn Reson Imaging 2000;11:174-181.

12. Manduca A, McGee KP, Welch EB, Felmlee JP, Grimm RC, Ehman RL. Autocorrection in MR imaging: adaptive motion correction without navigator echoes. Radiology 2000;215:904-909.

13. Loktyushin A, Nickisch H, Pohmann R, Schölkopf B. Blind multirigid retrospective motion correction of MR images. Magn Reson Med 2014;00:1-12.

14. Kober T, Marques J, Gruetter R, Krueger G. Head motion detection using FID navigators. Magn Reson Med 2011;66:135-143.

15. Pfeuffer J, Van de Moortele P-F, Ugurbil K, Hu XP, Glover G. Correction of physiologically induced global off-resonance effects in dynamic echo-planar and spiral functional imaging. Magn Reson Med 2002; 47:344-353.

16. Hu XP, Kim SGS. Reduction of signal fluctuation in functional MRI using navigator echoes. Magn Reson Med 1994; 31:495-503.

17. Splitthoff DN, Zaitsev M. SENSE shimming (SSH): a fast approach for determining $\mathrm{B}(0)$ field inhomogeneities using sensitivity coding. Magn Reson Med 2009;62:1319-1325.

18. Brau ACS, Brittain JH. Generalized self-navigated motion detection technique: preliminary investigation in abdominal imaging. Magn Reson Med 2006;55:263-270.

19. Jack CR, et al. Magnetic resonance imaging in Alzheimer's Disease Neuroimaging Initiative 2. Alzheimers Dement 2015;11:740-756.

20. Maclaren J. Measurement and correction of microscopic head motion during magnetic resonance imaging of the brain. PLoS One 2012;7: e48088.

21. Babayeva M, Kober T, Knowles B, Herbst M, Zaitsev M, Krueger G. Accuracy and precision of head motion information in multi-channel free induction decay navigators for magnetic resonance imaging. IEEE Trans Med Imaging 2015;34:1-11.

22. Herbst M, Maclaren J, Lovell-Smith C, Sostheim R, Egger K, Harloff A, Korvink J, Hennig J, Zaitsev M. Reproduction of motion artifacts for performance analysis of prospective motion correction in MRI. Magn Reson Med 2014;71:182-190.

23. Gedamu E, Gedamu A. Subject movement during multislice interleaved MR acquisitions: prevalence and potential effect on MRIderived brain pathology measurements and multicenter clinical trials of therapeutics for multiple sclerosis. J Magn Reson imaging 2012;36: 332-343.

24. Babayeva M, Loktyushin A, Falkovskiy P, Kober T, Mueli R, Gruetter R, Krueger G. FID navigator triggered acquisition of imaging navigators for retrospective head motion correction. ISMRM Workshop on Motion Correction, 2014, vol. 3703.

25. Falkovskiy P. Comparison of accelerated T1-weighted whole-brain structural-imaging protocols. NeuroImage 2016;124:157-167.

26. Reuter M, Tisdall MD, Qureshi A, Buckner RL, van der Kouwe AJW, Fischl B. Head motion during MRI acquisition reduces gray matter volume and thickness estimates. NeuroImage 2015;107:107-115.

27. Maréchal B, Kober T, Hilbert T, Ribes D, Chevrey N, Roche A, Thiran J-P, Meuli R, Krueger G. Automated quality control in MR-based brain morphometry. ISMRM 2012;2320:757.

28. Schmitter D, Roche A, Maréchal B, Ribes D, Abdulkadir A, BachCuadra M, Daducci A, Granziera C, Klöppel S, Maeder P, Meuli R, Krueger G. Alzheimer's Disease Neuroimaging Initiative. An evaluation of volume-based morphometry for prediction of mild cognitive impairment and Alzheimer's disease. NeuroImage Clin 2014;7:7-17.

29. Maréchal BM. Toward reliable MR-based brain structure morphometry: importance of rigorous quality control. École Polytechnique Fédérale de Lausanne, 2011.
30. Mortamet B, Bernstein MA, Jack CR, Gunter JL, Ward C, Britson PJ, Meuli R, Thiran J-P, Krueger G. Automatic quality assessment in structural brain magnetic resonance imaging. Magn Reson Med 2009; 62:365-372.

31. Kruggel F, Turner J, Muftuler LT, Alzheimer's Disease Neuroimaging Initiative. Impact of scanner hardware and imaging protocol on image quality and compartment volume precision in the ADNI cohort. NeuroImage 2010;49:2123-2133.

32. Gumus K, Keating B, White N, Andrews-Shigaki B, Armstrong B, Maclaren J, Zaitsev M, Dale A, Ernst T. Comparison of optical and MR-based tracking. Magn Reson Med 2015;74:894-902.

33. Alhamud A, Tisdall D, Hess AT, Hasan KM, Meintjes EM, van der Kouwe AJW. Volumetric navigators for real-time motion correction in diffusion tensor imaging. Magn Reson Med 2012;68:1097-1108.

34. Lin W, Nielsen T, Qin Q, Mostofsky SH, Wei J, Huang F, Duensing GR. Real-time motion correction in two-dimensional multislice imaging with through-plane navigator. Magn Reson Med 2014;71:1995-2005.

35. Dyverfeldt P, Deshpande VS, Kober T, Krueger G, Saloner D. Reduction of motion artifacts in carotid MRI using FID navigators. J Magn Reson Imaging 2014;40:214-220.

36. Babayeva M, Loktyushin A, Kober T, Granziera C, Nickisch $\mathrm{H}$, Gruetter R, Krueger G. FID-guided retrospective motion correction based on autofocusing. In Proceedings of the 22nd Annual Meeting of ISMRM, Milan, Italy, 2014. Abstract 2227.

37. Werner P, Barthel H, Drzezga A, Sabri O. Current status and future role of brain PET/MRI in clinical and research settings. Eur J Nucl Med Mol Imaging 2015;42:512-526.

38. Catana C, Benner T, van der Kouwe AJW, Byars L, Hamm M, Chonde DB, Michel CJ, El Fakhri G, Schmand M, Sorensen AG. MRI-assisted PET motion correction for neurologic studies in an integrated MRPET scanner. J Nucl Med 2011;52:154-161.

39. Keller SH, Hansen C, Andersen FL, Ladefoged C, Svarer C, Kjær A, Højgaard L, Law I, Henriksen OM, Hansen AE. Motion correction in simultaneous PET/MR brain imaging using sparsely sampled MR navigators: a clinically feasible tool. EJNMMI Phys 2015;2:14.

40. Babayeva M, Kober T, Herbst M, Hennig J, Seeger M, Gruetter R, Zaitsev M, Krueger G. Can multi-channel FID navigators quantify head motion? In Proceedings of the 21st Annual Meeting of ISMRM, Salt Lake City, Utah, USA, 2013. Abstract 306.

\section{SUPPORTING INFORMATION}

Additional Supporting Information may be found in the online version of this article.

Fig. S1. (a) Prescribed motion trajectory for the assessment of the registration reliability of the IMGnav. Artificial motion patterns for head nodding (nod), head shaking (shake), and moving the head in the head-foot direction (z-tra), and periods without motion, are depicted for rotation and translation in all six degrees of freedom. (b) Registration results from all subjects versus the applied motion trajectory for translation and rotation in $x, y$, and $z$ directions.

Fig. S2. Motion correction results from the data set A together with the detected motion trajectory for different subjects and motion patterns. An image acquired without any deliberate subject motion is shown for reference compared with the images with and without the motion correction mechanism in place. Here, the FID slow and FID fast were used for the detection of motion events: (a) Subject A.2 with z-translation motion pattern; (b) Subject A.4 with nodding motion pattern; (c) Subject A.5 with shaking motion pattern.

Table S1. Overview of All Acquired Data from All Subjects with the Corresponding Number of Detected Motion Events and the Extra Scan Time Needed for the Acquisition of the IMGnav and the Rescan of the Previous Train Echo, Which Is Considered Motion Corrupted (Also Provided Are the Maxima of the Detected Motion Together with the Results from Quantitative Image Quality Assessment through the Quality Index (QI) and Morphological Brain Segmentation Results for Total Intracranial Volume (TIV), Relative White Matter (WM), and Gray Matter (GM). 